\title{
Uso intensivo de herramientas y recursos de profesionalización política en campañas presidenciales: el caso de Colombia 2018
}

\section{Intensive use of tools and resources for political professionalization in presidential campaigns: the case of Colombia 2018}

\section{Uso intensivo de ferramentas e recursos para a profissionalização política em campanhas presidenciais: o caso da Colômbia 2018}

Nestor Julian Restrepo Echavarría, Universidad EAFIT, Medellín, Colombia (nrestr12@eafit.edu.co)

Luis Antonio González Tule, Universidad del Norte, Barranquilla, Colombia (ltule@uninorte.edu.co)

RESUMEN | Las campañas presidenciales en Colombia son cada vez más sofisticadas y profesionalizadas. Candidatos y partidos contratan expertos en distintas áreas, hacen un uso intensivo de las nuevas tecnologías y de los medios de comunicación, personalizan la campaña en el candidato y realizan sondeos de opinión o encuestas de percepción ciudadana. Considerando los avances en la profesionalización, los objetivos de este trabajo son, en primer lugar, medir el nivel de profesionalización delas campañas de los tres principales candidatos presidenciales de Colombia en 2018 (Iván Duque, Gustavo Petro y Sergio Fajardo), y, posteriormente, identificar las áreas de mayor desarrollo de técnicas e innovación que presentaron los diferentes grupos de dichas campañas. El nivel de profesionalización se midió a través de un índice construido en trabajos anteriores que permite hacer comparaciones internas y externas en las campañas. Los casos analizados ponen de manifiesto que la ventaja en las preferencias electorales no es suficiente para obtener el triunfo, sino que se requieren campañas con cierto nivel de profesionalización. Asimismo, se puede identificar en la campaña presidencial de 2018 que el nivel de profesionalización se encuentra explicado por la dimensión comunicativa, dependiendo de las estructuras mediáticas, la proyección de la imagen y la emotividad de la puesta en escena de la herramienta comunicativa.

PALABRAS CLAVE: campañas presidenciales; profesionalización de las campañas; índice de profesionalización; Colombia. 
ABSTRACT | The presidential campaigns in Colombia are increasingly sophisticated and professionalized. Candidates and parties hire experts, make intensive use of new technologies and the media, personalize the campaign, and conduct opinion polls or surveys regarding citizens' perception. Considering the professionalization advances, the objectives of this work are, first, to measure the level of the campaign's professionalization of the three main presidential candidates of Colombia in 2018 (Iván Duque, Gustavo Petro, and Sergio Fajardo), and, second, to identify the areas of greater development of techniques and innovation made by the different groups of these campaigns. To measure the level of professionalization, we built an index that allows comparisons inside and outside the campaigns. The cases analyzed show that the advantage in electoral preferences is not enough to obtain victory, but rather that campaigns with a certain level of professionalization are required. Likewise, we can see that in the 2018 Colombian presidential campaign the level of professionalization is explained by the communicative dimension, depending on the media structures, the image projection and the emotionality of the staging of the communicative tool.

KEYWORDS: presidential campaigns; professionalization of campaigns; professionalization index; Colombia.

RESUMO | As campanhas presidenciais na Colômbia estão se tornando mais sofisticadas e profissionalizadas. Os candidatos e partidos contratam especialistas em diversas áreas, fazem uso intensivo das novas tecnologias e dos meios de comunicação, personalizam a campanha sobre o candidato e realizam sondagens de opinião ou de percepção cidadã. Considerando os avanços na profissionalização, os objetivos deste trabalho são, em primeiro lugar, medir o nível de profissionalização das campanhas dos três principais candidatos presidenciais da Colômbia em 2018 (Ivan Duque, Gustavo Petro e Sergio Fajardo), e, em segundo lugar, identificar as áreas de maior desenvolvimento de técnicas e inovação apresentadas pelos diferentes grupos destas campanhas. Para medir o nível de profissionalização, construímos um índice que permite fazer comparações tanto dentro quanto fora das campanhas. Os casos analisados mostram que a vantagem nas preferências eleitorais não é suficiente para obter a vitória, senão que são necessárias campanhas com certo grau de profissionalização. Da mesma forma, pode-se identificar que na campanha presidencial de 2018 o nível de profissionalização se explica pela dimensão comunicativa, dependendo das estruturas midiáticas, da projeção da imagem e da emotividade da encenação da ferramenta comunicativa.

PALAVRAS-CHAVE: campanhas presidenciais; profissionalização das campanhas; índice de profissionalização; Colômbia. 


\section{INTRODUCCIÓN}

Cómo dice Cárdenas Ruiz, "las elecciones presidenciales de 2018 en Colombia se dieron en medio de un proceso de transición política en el marco del posconflicto donde estaba en juego la continuidad del Acuerdo de Paz y la tensión por nuevas agendas temáticas" (2020, p. 47). En este escenario de incertidumbre, las estrategias de comunicación política evidenciaron una mayor profesionalización a través del uso de nuevas tecnologías de la información, la personalización de la campaña en el candidato y no en el partido político, la contratación de asesores externos expertos en marketing político, imagen, redes sociales y producción audiovisual y en la realización de encuestas, grupos focales o sondeos. Frente a esto cabe preguntarse, ¿cuál es el nivel de profesionalización que desarrollaron las distintas campañas?, y ¿cómo es posible determinar el nivel de manera que pueda ser de utilidad para análisis comparados?

Este trabajo pretende dar respuesta a estos interrogantes y se propone medir el nivel de profesionalización de las campañas presidenciales de los tres candidatos más votados en Colombia: Iván Duque, del Partido Centro Democrático (39,14\% de votación), Gustavo Petro, de la coalición Colombia Humana (25,08\%) y Sergio Fajardo, del movimiento Compromiso Ciudadano (23,7\%). Esto a partir del Índice de Profesionalización (INPRO), el cual considera la estructura interna de la campaña y los recursos comunicativos de partidos y candidatos, y permite hacer comparaciones al interior y al exterior de las campañas.

Para ello, el texto está dividido en tres partes. La primera define conceptualmente la profesionalización de las campañas y ubica contextualmente el desarrollo de dicha profesionalización. La segunda parte lleva el concepto al plano operativo, de donde surge la herramienta analítica que servirá para estudiar las campañas, el índice basado en la observación de factores organizacionales y comunicativos de los partidos políticos. En una tercera sección se mide el nivel de profesionalización de las campañas de Iván Duque, Gustavo Petro y Sergio Fajardo, para después concluir con las principales reflexiones.

\section{LA PROFESIONALIZACIÓN DE LAS CAMPAÑAS}

La profesionalización hace referencia al proceso de evolución de las campañas electorales. Dicho proceso aparece como consecuencia, entre otros motivos, de los cambios en los medios de comunicación, así como cambios sociales y en los sistemas electoraly departidos (Holtz-Bacha, 2002). Eneste sentido, una campaña profesional es

aquella que utiliza tácticas y estrategias que privilegian el uso intensivo de medios de comunicación masivos, así como de las más recientes innovaciones tecnológicas para llegar a los votantes (media-intensive), las cuales son guiadas 
por sofisticados métodos de investigación de mercado y opinión pública (encuestas, grupos de discusión, bases de datos, investigación de la oposición, etcétera) (poll-driven) y planeadas por consultores profesionales, expertos en marketing político y en manejo de medios (consultant-driven) (Díaz 2015 pp. 125-126, con base en Farrell, 1996 y Smith 2004, 2009).

Con esta perspectiva, se pueden identificar tres etapas distintas en la evolución de la comunicación política, en especial en campañas electorales: las campañas premodernas, modernas y posmodernas. Dichas etapas muestran cómo se transforman simultáneamente la organización de la campaña, los medios de comunicación y el electorado, y cómo se informa del proceso electoral (Norris, 2002; Plasser \& Plasser, 2002; Blumler \& Kavanagh,1999).

La primera etapa en la evolución de las campañas se inicia en el siglo XIX y abarca hasta 1950. En esta, la comunicación política estuvo organizada desde los partidos políticos a nivel local y, dado que existía un fuerte vínculo partidista, el contacto con el electorado se realizaba de forma directa. Dentro de los medios de comunicación y canales de difusión destacan el uso de prensa partidista, la publicación de folletos y pega de afiches, la realización de mítines locales y, entrado el siglo XX, la difusión de spots a través de la radio (Gibson \& Römmele, 2001; LeDuc, Niemi, \& Norris, 2002).

Una segunda etapa, de 1960 a 1990, corresponde a las campañas modernas. A diferencia de la anterior, aquí la organización de la campaña se lleva a cabo desde el nivel central y los partidos políticos comienzan a ser asesorados por consultores externos, quienes se encargan de diseñar parte de la estrategia. A los medios de comunicación tradicionales se suma la televisión, que durante este periodo se convierte en el principal canal de promoción de partidos y candidatos. La consecuencia directa de esta nueva forma de recibir información fue el declive de la política cara a cara, así como la pérdida paulatina de vínculos partidistas (Gibson \& Römmele, 2001; LeDuc et al., 2002). Durante esta etapa comienza a hacerse uso de instrumentos de retroalimentación, como encuestas y grupos focales para conocer a la población.

La etapa actual corresponde a la de las campañas posmodernas. Esta da inicio en la década de 1990 y una de sus principales características es la presencia de consultores externos - expertos en medios, producción audiovisual, marketing político y comunicación política-, quienes tienen un rol mucho más influyente en el diseño de la estrategia y en su ejecución. Los mensajes y anuncios se vuelven más complejos, destacando la segmentación del electorado a partir del conocimiento de sus preferencias con instrumentos de medición y el uso de nuevas tecnologías -mensajes de texto, correos electrónicos y redes sociales-. Debido a la pérdida de identificación partidista y al distanciamiento candidato-elector de la etapa 
anterior, en las campañas posmodernas los partidos asignan mayor importancia a las organizaciones locales para celebrar sus actividades proselitistas (Gibson \& Römmele, 2001; LeDuc et al., 2002).

Dicho lo anterior, Holtz-Bacha (2007) concibe la profesionalización "como un proceso de adaptación y, como tal, una consecuencia necesaria de los cambios en el sistema político, por un lado, y en el sistema de medios, por otro, así como de la relación entre ambos sistemas" (p. 63). Por lo tanto, la profesionalización se refiere no solo a un alejamiento de las formas tradicionales de campaña electoral hacia formas y medios más modernos, sino también a cambios dentro del sistema político (Negrine, Mancini, Holtz-Bacha, \& Papathanassopoulos, 2007).

\section{ANTECEDENTES}

Colombia es uno de los países de la región donde ha existido mayor estabilidad de las instituciones, fundamentada en la continuidad de sus procesos electorales, a pesar de haber tenido un conflicto armado. En este contexto, las campañas políticas en el país se han reconocido por desarrollarse dentro de un espacio ideológico bipartidista, en el que los partidos Liberal y Conservador se enfrentaban en la escena política para lograr el poder público y en el que la propaganda política, los discursos masivos, la radio y la prensa se convirtieron en los elementos comunicativos más importantes para persuadir al elector.

Como consecuencia de la baja institucionalidad del Estado colombiano, de la falta de credibilidad y convicción ideológica hacia los partidos políticos, la globalización y los nuevos mecanismos de participación ciudadana, en conjunto con las nuevas herramientas mediáticas, se han evidenciado cambios en los métodos empleados para desarrollar las campañas electorales (Restrepo-Echavarría, 2015). Según Restrepo-Echavarría "las campañas presidenciales en Colombia han migrado paulatinamente de las calles y plazas públicas a los debates de televisión, y de los mítines políticos a los foros virtuales en redes sociales, en las que se deliberan los temas de la agenda pública, como el proceso de paz y el narcotráfico, sobrepasando otros como el desempleo, la corrupción y la economía" (2015, p. 88).

Con la promulgación de la Constitución política de 1991, se modificó el sistema electoral con efectos sobre el sistema partidista: se establecieron las reglas que permitirían la segunda vuelta para la elección presidencial, buscando dotar de mayor legitimidad al candidato ganador; se introdujeron cambios en el esquema de financiación de las campañas, tomando vigencia el sistema de financiación mixta, y se potenciaron las campañas subnacionales a partir de la elección de alcaldes y gobernadores (Restrepo-Echavarría, 2017). 
En el sistema de partidos, se pasó de un sistema bipartidista a uno multipartidista, ampliando la participación política a diferentes grupos sociales (indígenas, raizales, cristianos, protestantes). Esto contribuyó a que los partidos políticos pasaran de tener una estructura interna basada en facciones ideológicas e institucionalizadas a fundamentarse en personalismos políticos.

En efecto, Pizarro (2008) evidencia que el sistema de partidos colombiano empezó a materializar síntomas de desinstitucionalización, específicamente en dos dimensiones: la legitimidad de las organizaciones políticas y la solidez organizativa de las estructuras internas. A raíz de esto, surgen las candidaturas independientes, campañas concebidas por el candidato, al igual que su financiación y sin la tutela del partido político, cuya función se limitará a prestar su nombre y ofrecer un aval para oficializar las candidaturas (Pizarro, 2008).

En las elecciones presidenciales de 2018 se amplía el fenómeno de la personalización. Estas elecciones consolidaron el fortalecimiento de las marcas políticas personales y el hundimiento de las formaciones partidistas tradicionales. En este sentido, las figuras de Gustavo Petro y Sergio Fajardo se fortalecieron como referentes de centroizquierda tras cosechar un apoyo histórico en las urnas que, pese a no otorgarles la presidencia, sitúa a la izquierda como una alternativa real y viable de poder tras décadas de ostracismo. Mientras que en la campaña de Iván Duque, a la postre ganador de la contienda, la personalización recayó en la imagen del expresidente y fundador del Partido Centro Democrático, Álvaro Uribe.

\section{METOdOLOGÍA}

La herramienta analítica para medir el nivel de profesionalización de las campañas colombianas adopta criterios propuestos por Gibson y Römmele (2001, 2009) y Strömbäck (2009). Para la construcción de su índice, Gibson y Römmele (2001,2009) plantean observar diez variables para captar los elementos básicos de una campaña, tomando en consideración las herramientas y estrategias empleadas, el modo en el que se usan y la naturaleza de las relaciones de poder al interior de la campaña. En un primer trabajo (Gibson \& Römmele, 2001) el índice adopta criterios dicotómicos - presencia o ausencia del fenómeno-, mientras que más adelante clasifican las variables a observar en subjetivas y objetivas, y miden no la presencia o ausencia sino la intensidad en que se presenta el fenómeno a través de una escala de 0 a 3.

Una revisión a esta propuesta la elabora Strömbäck (2009), quien considera que Gibson y Römmele construyeron un modelo centrado en el partido (party-centred theory) y prestaron poca atención a otros elementos de la profesionalización de las 
campañas. Strömbäck sugiere considerar además del uso de nuevas tecnologías y la presencia de expertos en el manejo de campañas, el uso de técnicas sofisticadas, dentro de las que destaca la organización de grupos focales. Al igual que Gibson y Römmele (2009), cuando reformulan su índice, Strömbäck (2009) crea variables con tres categorías. Para este autor, su índice captura mejor la profesionalización en sistemas multipartidistas a diferencia del índice de Gibson y Römmele, que se adapta más a sistemas bipartidistas.

Dado que las técnicas y los instrumentos de campaña continuarán evolucionando a lo largo del tiempo y de acuerdo con el contexto (Strömbäck, 2009), los criterios para medir la profesionalización también deben irse modificando. Con esta consideración, distintos autores han adaptado los índices anteriores para medir la profesionalización de algunas campañas en América Latina. De esta manera, Rocha (2007) analiza las campañas presidenciales en Brasil de 1989 a 2006. El caso colombiano sobre las campañas presidenciales de 2010 y 2014 es abordado por Restrepo-Echavarría (2015) y Restrepo-Echavarría, Rodríguez y Castromil (2018), respectivamente. Por otra parte, González y Restrepo-Echavarría (2020) miden el nivel de profesionalización de la campaña presidencial mexicana de 2018.

Siguiendo a todos estos autores, el índice de profesionalización aquí propuesto (INPRO) se compone de dos dimensiones. La dimensión organizativa identifica la estructura interna de la campaña, las personas que en ella participan y la manera en la que se organizan. Esta se encuentra dividida en tres categorías: estratégica, táctica y técnica (Restrepo-Echavarría et al. 2018; González \& RestrepoEchavarría, 2020). A continuación, se detallan los indicadores y valores de la dimensión organizativa.

1. Comando de las campañas. El argumento que se esgrime en la literatura es que, a mayor centralización del comando de toma de decisiones de la campaña, mayor profesionalización. Cuando el comando de toma de decisiones de la campaña es descentralizado, el candidato, partido o coalición adquiere un valor de 0; cuando el comando es semi centralizado adquiere 1, y cuando es centralizado, 2.

2. Consultores externos. La presencia de consultores externos al partido es uno de los rasgos principales de la profesionalización (Holtz-Bacha, 2002). El valor 0 se asigna cuando la campaña no tuvo consultor profesional externo en ningún área; el 1, cuando el consultor profesional externo participa de manera eventual durante la campaña y se encarga de funciones determinadas, y el 2, cuando se evidencia la participación del consultor profesional externo durante toda la compaña. 
3. Gerenciamiento profesional dela campaña. A mayor experiencia y especialización en la coordinación de la campaña, mayor profesionalización. Se asigna un valor de 0 cuando la campaña es coordinada por personal del partido sin experiencia previa en campañas electorales; un 1 cuando la campaña es coordinada por personal del partido con experiencia en campañas electorales, y 2, cuando la campaña es coordinada por gerente(s) profesional(es) ajeno(s) al partido político o al candidato.

4. Campaña permanente. Siguiendo a Gibson y Römmele (2001, 2009), una de las características de la profesionalización es que las campañas son permanentes. De acuerdo con esto, si en la campaña el candidato limita su estrategia de comunicación a los tiempos establecidos por el sistema electoral adquiere el valor 0; si el candidato trata de generar estrategias de comunicación, tanto en los momentos en los que compite por la nominación como fuera de estos, pero el sistema electoral limita los ciclos de campaña, adquiere 1, y si el candidato trata de generar estrategias de comunicación, tanto en los momentos en los que compite por la nominación como fuera de estos, y no hay límites efectivos que establezca el sistema electoral, adquiere 2 .

5. Personalización de la campaña en el candidato. La literatura coincide en señalar que cuanto más personalizada es la campaña en el candidato, mayor es la profesionalización. Se asigna el valor 0 cuando el partido y líderes partidistas son el foco de la campaña; 1, cuando la campaña combina la promoción del candidato junto con la del partido, y 2, cuando el foco de la campaña está centrado en el candidato.

6. Profesionalización del grupo de campaña. Desde la campaña de Bill Clinton se popularizó la creación de grupos de campaña especializados en distintas áreas (Strömbäck, 2009). Se asigna 0 cuando el candidato y sus colaboradores realizan todos los trabajos en la campaña; 1, cuando algunas áreas de la campaña están manejadas por personas expertas en el tema y el resto del personal son colaboradores, y 2 , cuando el grupo está conformado por áreas de trabajo y en cada una hay personal profesional de expertos.

7. Militancia remunerada de la campaña. El pago de servicios está asociado a la especialización del trabajo realizado. Adquiere un valor de 0 cuando todos los colaboradores en la campaña son voluntarios; 1 , cuando hay una mezcla entre voluntarios y personal pagado en la campaña, y 2, cuando todo el personal de la campaña recibe un salario por su labor.

8. Financiación. Este indicador toma en cuenta los mecanismos y formas para la consecución de la financiación de la campaña, considerando que en Colombia 
se permite el financiamiento privado. Se califica con 0 si la campaña no tiene un área dedicada a la consecución y destinación de los recursos financieros (sin Polítical Action Commitees, PAC), sino que la consecución de fondos recayó sobre el gerente de campaña o el candidato; 1 , si la campaña tuvo un gerente y un grupo del partido dedicado a la consecución de recursos, y 2 si la campaña contó con un comité de especialistas externos al partido dedicado a la consecución y destinación de los recursos financieros (con PAC).

9. Monitoreo electoral. El uso de herramientas de monitoreo es esencial en una campaña para conocer y segmentar al electorado y adaptar la estrategia (Strömbäck, 2009). Se asigna el valor 0 cuando la campaña no usa encuestas, sondeos ni grupos focales; 1 , cuando la campaña interpreta las encuestas públicas y esporádicamente contrata expertos de encuestas de opinión, y 2 cuando hace uso de sondeos, encuestas o grupos focales y tiene personal experto en análisis de estos instrumentos de monitoreo.

La segunda dimensión, denominada comunicativa, capta las estrategias y los recursos comunicacionales de los partidos y candidatos. Esta también se subdivide en tres categorías: discursiva, procesos de comunicación y medios técnicos (Restrepo-Echavarría et al., 2018; González \& Restrepo-Echavarría, 2020). Los indicadores y valores de la dimensión comunicativa se detallan a continuación.

1. Mensajes personalizados. Cuando se evidencia que los mensajes se centran en el partido y no en el candidato se asigna el valor 0; cuando los mensajes intercalan al candidato con el partido, se asigna 1, y 2 cuando se caracterizan por tener un alto enfoque hacia el candidato por encima del mensaje del partido y la ideología.

2. Uso de referentes simbólicos. Si el contenido es directo y visible el interés solo de informar adopta el valor 0; si el mensaje combina contenido informativo y simbólico, se asigna 1, y si el mensaje tiene un alto contenido simbólico, adopta 2 .

3. Segmentación de los mensajes. Cuando en la campaña solo hay mensajes estándar invitando a votar por el candidato, adquiere el valor 0; cuando la campaña divide los públicos, pero comunica los mismos mensajes, se asigna 1, y 2 cuando hay un alto nivel de fragmentación de los mensajes, tanto por públicos, como diferentes tipos de mensajes según el target.

4. Uso emocional de los mensajes electorales. Cuando los contenidos de los mensajes son directos y estándar, se asigna 0; adopta el valor 1 cuando el contenido de algunos mensajes genera emoción, y 2 cuando los mensajes tienen un alto nivel emocional. 
5. Construcción de imagen. Se asigna 0 cuando la imagen física y discursiva del candidato no es preponderante por la falta de acompañamiento; 1 , cuando los estrategas del partido se encargan de construir una imagen física y discursiva del candidato, y 2 , cuando el candidato cuenta con un grupo de profesionales encargado de la construcción de su imagen pública y le construye su discurso político.

6. Construcción de una narrativa (Storytelling). Mediante el lenguaje y la retórica, los candidatos construyen una narrativa personal para movilizar al electorado. La estrategia narrativa suele ser autobiográfica o familiar (Liebhart \& Bernhart, 2017). Si el candidato no desarrolla una estrategia discursiva que construya relatos, se asigna 0; recibe 1 cuando el candidato construye relatos pero sin preparación y con un alto nivel de improvisación, y si el candidato construye una narrativa para movilizar al electorado, adquiere el valor 2 (Restrepo-Echavarría et al., 2018).

7. Participación en debates $y$ foros. La participación en debates y foros guarda relación con la profesionalización, pues requiere un trabajo especializado de asesoramiento para preparar la imagen del candidato, el manejo de cámara, su discurso, etc. Cuando el candidato no participa en debates ni foros como programas televisivos (de distinta índole, incluso cómicos) o en plataformas virtuales (debatiendo con youtubers, por ejemplo), se asigna 0; recibe 1 cuando la participación en debates o foros es esporádica, y cuando participa en todos los foros y debates, se asigna 2.

8. Material escrito para uso externo en las campañas electorales. Si en la campaña es inexistente el uso de publicidad escrita, o es incipiente con algún material de volantes, adquiere el valor 0; cuando la campaña usa todo tipo de material escrito, pero sin acceso a vallas publicitarias por su alto costo, el 1, y 2 cuando hay en la campaña un uso masivo de vallas, publicidad externa y vallas móviles, material de prensa y volantes acompañados de una estrategia de señalética.

9. Uso de nuevos recursos: WhatsApp, Twitter, YouTube, Facebook, Instagram, SMS. Se asigna 0 cuando en la campaña no se hace uso de este tipo de recursos; 1 cuando se hace uso frecuente de dichos recursos, y 2 cuando se identifica su uso a lo largo de toda la campaña.

El índice de profesionalización (INPRO) se obtiene promediando el resultado de las dos dimensiones de análisis. En cada dimensión se suman los valores de sus indicadores y luego se divide por el número total de indicadores. La fórmula para calcular el INPRO se muestra a continuación: 


\section{Índice de profesionalización (INPRO)} Dimensión Organizativa: $I N P R O o=\frac{\sum \text { intensidad indicadores Dimensión Organizativa }}{10}$

Dimensión Comunicativa: $I N P R O c=\frac{\sum \text { intensidad indicadores Dimensión Comunicativa }}{9}$

Índice de Profesionalización: INPRO =

INPROO+INPROC

2

Fuente: Elaboración propia con base en Restrepo-Echavarría et al., 2019.

\begin{tabular}{ccc}
$\begin{array}{c}\text { Índice de profesionalización } \\
\text { (INPRO) }\end{array}$ & $\begin{array}{c}\text { Nivel de } \\
\text { profesionalización }\end{array}$ & \% de profesionalización \\
\hline $0 \leq$ INPRO $<0,5$ & Bajo & $0 \% \leq$ INPRO $<25 \%$ \\
\hline $0,5 \leq$ INPRO $<1$ & Medio & $25 \% \leq$ INPRO $<50 \%$ \\
\hline $1 \leq$ INPRO $<1,5$ & Medio-alto & $50 \% \leq$ INPRO $<75 \%$ \\
\hline $1,5 \leq$ INPRO $\leq 2$ & Alto & $75 \% \leq$ INPRO $\leq 100 \%$ \\
\hline
\end{tabular}

Tabla 1. Nivel de profesionalización de las campañas electorales

Fuente: Elaboración propia con base en Rocha (2007).

Con el valor del INPRO se podrá saber el nivel de profesionalización que adopta cada campaña, tal y como se muestra en la tabla 1.

Las fuentes de información provienen del seguimiento de medios de comunicación durante y después de las campañas, del análisis de los spots publicitarios en televisión y radio, de las páginas oficiales de los candidatos y partidos, así como de entrevistas a colaboradores y consultores políticos de las tres campañas electorales. Se realizaron entrevistas entre los meses de abril y mayo de 2019 a los coordinadores regionales de la campaña presidencial de Iván Duque, Mario Hernández y Juan Fernando Jaramillo; al asesor de la campaña de Gustavo Petro, el argentino Ángel Beccassino, y a los consultores Miguel Jaramillo Lujan, director de MPG, y Nury Astrid Gómez Serna, directora de la empresa de consultoría Máximo Impacto, con quienes se abordó la campaña de Sergio Fajardo. 


\section{RESULTADOS}

\section{Dimensión organizacional}

Comenzando con el indicador Comando de las campañas, la característica principaldelas campañas presidenciales colombianas es quefueron descentralizadas o semi centralizadas (tabla 2). La campaña de Duque tuvo una parcial centralización del comando con una gerencia y un grupo central de campaña dirigidos desde Bogotá. Se pudo identificar una campaña descentralizada donde cada grupo, por regiones y por municipio, generó la estrategia tanto en la publicidad como en los mensajes y eventos del candidato. De hecho, uno de los problemas que tuvo la campaña fue la financiación de los grupos, que no recibieron dinero del grupo central para elaborar la estrategia de campaña.

La campaña de Petro tuvo un comando parcialmente centralizado, conformado en su mayoría por comités de ayuda y grupos de seguidores en las regiones, principalmente jóvenes y estudiantes, quienes cumplieron la función de realizar la campaña al interior del país. Estos estuvieron acompañados por el grupo central de la campaña, que manejaba la agenda del candidato en su gira por distintas regiones. La campaña de Fajardo tuvo un comando descentralizado, dejando la estrategia de marketing político nacional a unos pocos integrantes de la campaña que han pertenecido a su grupo de origen Compromiso Ciudadano.

En relación con la presencia de consultores externos dentro de las campañas, la campaña de Duque no contó con un asesor externo, tan solo en la segunda vuelta tuvo una esporádica ayuda del publicista Carlos Alberto Cortés, gerente en Do Consulting, quien se encargó de construir la estrategia discursiva de la campaña. Se podría decir que la campaña en su parte estratégica estuvo dirigida por su gerente de campaña Luis Guillermo Echeverri, hijo de Fabio Echeverri, integrante de las campañas electorales de Álvaro Uribe Vélez. Por su parte, Petro contó con el apoyo del asesor externo argentino Ángel Beccassino, quien fue el encargado de la publicidad y en general de la asesoría de movilización de la campaña. De igual manera, en la segunda vuelta fue fundamental en la construcción del mensaje de esperanza y del ataque frontal al miedo generado por el candidato del Centro Democrático. La campaña de Fajardo no contó con asesor externo, tan solo con el apoyo de personas muy experimentadas en el tema electoral que han estado en sus anteriores campañas, tanto para la alcaldía de Medellín como para la gobernación de Antioquia.

Del indicador gerenciamiento profesional, cabe señalar que la campaña de Duque contó con un grupo de colaboradores cercanos al gobierno de Álvaro Uribe y con amigos personales del candidato. Dicho grupo estuvo dirigido Luis Guillermo Echeverri. Al frente de la estrategia de comunicación se encontraron 


\begin{tabular}{|c|c|c|c|c|}
\hline Categoría & Indicador & Duque & Petro & Fajardo \\
\hline \multirow{5}{*}{ Estratégica } & $\begin{array}{c}\text { Comando de las campañas: centralizado vs. } \\
\text { descentralizado. }\end{array}$ & 1 & 1 & 0 \\
\hline & $\begin{array}{c}\text { Presencia de consultores externos en áreas de } \\
\text { comando. }\end{array}$ & 0 & 2 & 0 \\
\hline & Gerenciamiento profesional de campañas. & 2 & 1 & 1 \\
\hline & Campaña permanente. & 0 & 2 & 2 \\
\hline & $\begin{array}{c}\text { Personalización de la campaña en el candidato, } \\
\text { alejándose de los programas y orientaciones } \\
\text { ideológicas del partido. }\end{array}$ & 1 & 2 & 2 \\
\hline \multirow{3}{*}{ Táctica } & $\begin{array}{l}\text { Profesionalización del grupo de campaña, un grupo } \\
\text { de trabajo multi e interdisciplinario. }\end{array}$ & 2 & 1 & 1 \\
\hline & Militancia remunerada en la campaña. & 1 & 1 & 1 \\
\hline & Financiación. & 0 & 1 & 0 \\
\hline \multirow[t]{3}{*}{ Técnica } & $\begin{array}{l}\text { Monitoreo electoral: uso de sondeos, encuestas y } \\
\text { grupos focales. }\end{array}$ & 1 & 1 & 1 \\
\hline & Media training y entrenamiento de equipo. & 2 & 2 & 2 \\
\hline & INPRO & 1,0 & 1,4 & 1,0 \\
\hline
\end{tabular}

Tabla 2. Dimensión organizacional

Fuente: Elaboración propia.

Gloria Ramírez y Juan Pablo Bieri, periodistas con gran experiencia en medios de comunicación a nivel nacional. La estrategia en campo la desempeñó su jefa de debate, Alicia Arango, de gran tradición en el partido y una persona leal de Álvaro Uribe en su presidencia. Otra integrante de la campaña muy cercana al candidato fue María Paula Correa, su asistente personal, quien trabajó en los dos periodos de la presidencia de Álvaro Uribe.

La campaña de Petro se distinguió por un mediano gerenciamiento, ya que en su mayoría eran personas que siempre han estado con el candidato desde la alcaldía de Bogotá, como Holman Morris, jefe de debate, María Maldonado, jefa de programación de campaña, Jorge Rojas, estratega interno de la campaña, Augusto Rodríguez, gerente, y finalmente, voluntarios en todo el país. De igual manera se puede evidenciar un nivel de profesionalización medio en Fajardo, ya que tanto su gerente como su grupo de campaña más importante adquirieron experiencia en los diferentes cargos que ocuparon en el departamento de Antioquia.

En cuanto al tiempo a lo largo del cual hicieron campaña, destaca que el abanderado del Centro Democrático, Iván Duque, no realizó una campaña permanente pues, a diferencia de muchos candidatos que han tenido una amplia 
carrera política, Duque no tenía experiencia ni tradición partidista. Para el analista político Gilberto Tobón Sanín, "Duque fue una fabricación del líder del partido Álvaro Uribe Vélez, quien con el comité del partido Centro Democrático lo eligió como candidato presidencial por encima de otros experimentados candidatos" (Nos cogió la noche Cosmovisión, 2018, 1:41).

Por su parte, Petro y Fajardo hicieron campaña permanente, desde la gestión como gobernantes en las alcaldías de Bogotá y Medellín, respectivamente, el Senado de la Republica - para Petro - y la gobernación del departamento de Antioquia - para Fajardo-, donde a pesar de las limitaciones legislativas que prohíben la participación en política (Procuraduría General de la Nación, 2017) ambos construyeron su imagen y consignas de gobiernos. En el caso de Petro fue Bogotá Humana, que en la campaña nacional se llamaría Colombia Humana; en el de Fajardo, fue Antioquia la más educada, que le daría toda la plataforma a su campaña presidencial como profesor para la presidencia de Colombia. De igual manera, ambos construyeron su discurso a favor de un cambio y en contra de la clase política tradicional y de la corrupción.

Con respecto a la personalización de las campañas, el caso de Duque podría calificarse como medianamente personalizado, pues, aunque se trató de construir la imagen de un candidato que era desconocido para la mayoría de la opinión pública, era el que tenía el apoyo del líder del partido, Álvaro Uribe. De igual manera, esta campaña contó con el apoyo sustancial en la generación de mensajes y ataques a los otros candidatos y a los electores por parte de los integrantes del Centro Democrático. Las campañas de Petro y Fajardo fueron campañas altamente personalizadas, pues al no contar con un acompañamiento de los partidos tradicionales, buscaron posicionar sus liderazgos por encima de los colectivos partidistas.

En relación con la profesionalización del grupo de campaña a través de un grupo de trabajo multi e interdisciplinario, la campaña de Duque evidencia el nivel más alto de profesionalización (¿A quiénes escuchan...?, 2018). Este candidato tuvo colaboración de personal de diferentes áreas y colaboradores aliados que habían tenido una amplia experiencia en el gobierno de Álvaro Uribe. Dicho grupo estuvo estructurado en tres equipos multidisciplinarios, coordinados por su gerente de campaña Luis Echeverri. El primero, su círculo cercano, integrado por políticos del Centro Democrático, empresarios y personal experto en temas de comunicación política. El segundo, integrado por un grupo de comunicadores y periodistas que siguieron al candidato y recogieron las declaraciones, imágenes y videos de sus recorridos. Por último, un equipo conformado por unos 15 millennials, que se encargó de los contenidos para Instagram, Facebook y WhatsApp (Gómez, 2018). 
La campaña de Petro tuvo un mediano nivel de profesionalización pues, aunque fue un grupo con experiencia, no son en su mayoría personas con alto bagaje y promoción en sus profesiones. Su gerente de campaña fue Augusto Rodríguez, amigo del candidato quien dirigió un grupo de colaboradores que estuvo acompañando a Petro desde su gobierno en la alcaldía de Bogotá. De igual manera, Petro estuvo apoyado por las bases sociales y grupos de presión de la izquierda colombiana, como el sindicato de FECODE (sindicato de profesores de Colombia) y estructuras de los partidos Unión Patriótica y parte del Polo Democrático y el MOIR. Finalmente, la campaña de Fajardo contó con el apoyo de personas expertas en temas empresariales, en especial el grupo empresarial más importante del país: el grupo empresarial antioqueño. Sus colaboradores directos fueron los que siempre lo han acompañado en su proyecto político de Compromiso Ciudadano, parte de su grupo de trabajo de la alcaldía de Medellín y la Gobernación de Antioquia, más una gran base de colaboradores no remunerados.

En cuanto a la militancia remunerada de la campaña, dentro de la de Duque son muy pocas las personas en cargos específicos que tuvieron reconocimiento salarial. En su gran mayoría, la campaña estuvo compuesta por grupos de colaboradores, muchos de los cuales pertenecían a su vez a otros grupos, en especial personal de los congresistas de la República, colaboradores del uribismo en las regiones y municipios. Se debe considerar que, tanto en la campaña de Petro como en la de Fajardo, muchos de estos grupos en sus territorios financiaban ellos mismos la publicidad y los diferentes insumos que exigía el rigor de la campaña. Otros eran grupos de seguidores y de personas que acompañaron las campañas ad honorem.

En el ámbito de la financiación de los partidos, candidatos y campañas electorales, se encontró que las campañas electorales no contaron con áreas estratégicas que se dedicaran a la consecución de fondos y destinación de los recursos financieros. De hecho, lo que se observa es el apoyo directo de las élites económicas. Por ejemplo, Luis Carlos Sarmiento Angulo, el hombre más rico de Colombia, financió más del 50\% de la campaña de Duque ${ }^{1}$, mientras que el grupo de los empresarios de Antioquia financió en parte la campaña de Fajardo. Finalmente, es importante resaltar que en este rubro la campaña de Petro contó con una gestión media de profesionalización, puesto que para la campaña recaudó fondos a partir de su portal de donaciones en su página web, donde, según información de varios periódicos, se logró facilitar el recaudo de dinero en especial de académicos y estudiantes universitarios (Segrera, n.d.).

1. Según el periódico el Espectador (El grupo Aval financió..., 2018), el grupo Aval financió el $66 \%$ de la campaña de Iván Duque. 
Por último, para concluir esta dimensión, respecto del uso de sondeos, encuestas, grupos focales para el seguimiento de las percepciones y diseño de la estrategia comunicativa, se evidenció un alto nivel de profesionalización en las campañas de Duque, Petro y Fajardo. Esta campaña presidencial se basó más en la construcción de escenarios emocionales que, en procesos de construcción de propuestas de campaña; de hecho, se evidencian más estrategias de comunicación política y técnicas de marketing político aprovechando la polarización electoral que había dejado el Plebiscito por la paz del 2 de octubre de 2017.

\section{Dimensión comunicativa}

En las tres campañas se puede evidenciar cómo la dimensión comunicativa es la más relevante a partir del uso de las diferentes técnicas y procesos de comunicación. La característica es la construcción del mensaje personalizado de los candidatos, en sus discursos en primera persona, cercanos a los ciudadanos, desmarcándose del partido político ${ }^{2}$. De igual manera, las campañas presidenciales tuvieron un alto uso de referentes simbólicos, cuyo objetivo era estimular el voto de los electores. En esta campaña, fue común la indignación, la inconformidad y el miedo al proceso de paz, la polarización ideológica que dejó el conflicto armado, la crisis venezolana y la confrontación política entre el expresidente Álvaro Uribe y el presidente saliente, Juan Manuel Santos.

Duque fue el candidato que más segmentó sus mensajes para tratar de llegar a distintos públicos. En su intención de atraer un electorado más joven en algunos anuncios se mostraba como un hombre alegre y rumbero, mientras que, ante otros sectores, como adultos y la población más conservadora, se mostró como un estadista, hombre educado y con carácter (Comitia Consulting, 2018). Petro, a diferencia de Duque, no usó ese tipo de segmentación. La mayor parte de su publicidad fue diseñada para cautivar al público joven y a los grupos y colectivos sociales inconformes con la situación política y económica del país con la propuesta del cambio (En Movimiento. Información y prensa, 2018). De igual forma, Fajardo segmentó medianamente sus mensajes construyendo el ideal del profesor honesto y sincero con los electores, buscando construir puentes entre todos los actores de poder $^{3}$ (Sergio Fajardo, 2018).

2. En las tres campañas se identificaron mensajes altamente personalistas, por ejemplo, en las vallas publicitarias: Duque: "No quiero vivir como venezolano. Duque Presidente, el futuro es de todos"; Fajardo: “Fajardo presidente, jse puede!"; Petro apelando a la construcción del Caudillo Jorge Eliecer Gaitán: "Petro presidente. No soy un hombre, soy un pueblo".

3. El profesor presidente es su slogan, que lo define como un progresista de centro, dejando de lado la polarización política que para ese momento marcaba la publicidad de la campaña presidencial. 


\begin{tabular}{|c|c|c|c|c|}
\hline Categoría & Indicador & Duque & Petro & Fajardo \\
\hline \multirow{6}{*}{ Discursiva } & Mensajes personalizados. & 2 & 2 & 2 \\
\hline & Uso de referentes simbólicos. & 2 & 1 & 1 \\
\hline & Segmentación de los mensajes. & 2 & 1 & 1 \\
\hline & Uso emocional de los mensajes. & 2 & 2 & 2 \\
\hline & Construcción de imagen y posicionamiento del candidato. & 2 & 2 & 2 \\
\hline & Storytelling. & 2 & 1 & 2 \\
\hline $\begin{array}{l}\text { Procesos de } \\
\text { comunicación }\end{array}$ & Participación en debates televisados y foros sociales. & 1 & 2 & 2 \\
\hline \multirow{3}{*}{$\begin{array}{l}\text { Medios } \\
\text { técnicos }\end{array}$} & $\begin{array}{c}\text { Material escrito para uso externo en las campañas } \\
\text { electorales. }\end{array}$ & 2 & 2 & 2 \\
\hline & $\begin{array}{c}\text { Uso de nuevos recursos: WhatsApp, Twitter, YouTube, } \\
\text { Facebook, Instagram, SMS. }\end{array}$ & 2 & 2 & 2 \\
\hline & INPRO & 1,88 & 1,66 & 1,77 \\
\hline
\end{tabular}

Tabla 3. La dimensión comunicativa

Fuente: Elaboración propia.

En relación con el uso emocional de los mensajes electorales, las campañas tuvieron un alto contenido, donde la división política por el Plebiscito del 2 de octubre de 2017 sobre los acuerdos de paz de La Habana había dejado una alta polarización y un ambiente de conflicto y puntos de vista divergentes. Las tres campañas construyeron mensajes emocionales: miedo, enojo y esperanza. Un ejemplo fue la campaña de Duque, donde su fórmula vicepresidencial Marta Lucia Ramírez se tomó una foto, visitando un supermercado en Venezuela con los anaqueles vacíos, como señal del futuro de Colombia en caso de un posible triunfo de Petro. La campaña de Petro usó un mensaje más directo y específico, con temas económicos y políticos de activación política, donde lo primordial era mostrar las falencias del Estado colombiano y recordarle a la opinión pública los actos de corrupción de los gobiernos anteriores. Por su parte, Fajardo pretendió ser el candidato neutral, el conciliador, buscando deslindarse de la clase política tradicional, estructurando un mensaje de educación y reconciliación.

En la categoría procesos de comunicación se evidencia la intención de construir y posicionar la imagen de los candidatos. En Duque, un candidato poco conocido, sin experiencia política y tradición partidista, la estrategia de comunicación política estuvo cargada de símbolos y signos. Se mostró como un candidato maduro y decidido en su intención de llegar a un electorado más adulto y conservador, pero también jovial y alegre, jugando fútbol o tocando instrumentos musicales, para 
llegar a un electorado más joven; la campaña construyó una imagen de estadista, de estudioso y el más indicado para seguir las ideas de Álvaro Uribe Vélez (Iván Duque presidente, 2018).

Petro, en cambio, efectuó su posicionamiento mediante un discurso apaciguador, para cambiar la percepción de la izquierda armada, cuya imagen en Colombia ha sido desprestigiada por décadas, a la vez que emprendió un discurso crítico frente al establecimiento. Su estrategia de posicionamiento fue mediante un discurso más socialdemócrata moderado, resaltando su experiencia previa como gobernante y posicionando temas como la corrupción y el apoyo al campo y a la agricultura (Julián Duque García, 2018).

De igual manera, es notoria la técnica del relato (storytelling) en las campañas de Duque y Fajardo, tanto en la construcción de la imagen como en la búsqueda del reconocimiento en todos los ciudadanos del país. Asimismo, en la construcción del mensaje político se hizo alusión a principios nacionalistas, apelando al origen y tradición de su ascendencia paisa - en el caso de Fajardo-, la construcción de imaginarios de honorabilidad y rectitud, la idea de ser personas justas, amables y sencillas. En la campaña de Petro no hubo claridad sobre la preparación de su relato, pues este candidato tuvo que sortear y defenderse del imaginario colectivo de los colombianos sobre el descrédito de las guerrillas colombianas y de los políticos de izquierda por la historia del conflicto armado.

Otro ítem fundamental de la dimensión comunicativa en las campañas presidenciales fue la amplia participación en los debates televisados. En esta campaña, se usaron diferentes formatos de debate dependiendo del grupo de medios de comunicación a nivel nacional y regional (Grupo RCN, Grupo Caracol TV, y canales regionales como Tele Antioquía, Tele Pacífico, Tele Caribe, que realizaban el debate. Se debe precisar que, en Colombia, los debates televisados son libres, no tienen regulación en número por parte del Consejo Nacional Electoral (CNE), y el candidato puede determinar si asiste o declina la invitación.

Dicha libertad en el número de debates hizo que en la campaña se realizarán más de 30 encuentros, entre debates televisados, en universidades, con gremios, instituciones y colectivos y hasta youtubers (Debates presidenciales, ¿es momento de regularlos?, 2018). El único candidato que rehusó acudir al llamado de youtubers fue Iván Duque, quien también se negó asistir a debates en la segunda vuelta. De hecho, se evidenció una mejor preparación de Petro y Fajardo en el manejo de la cámara, en el entrenamiento de la expresión oral y corporal para resolver las preguntas, y en la preparación sobre diferentes temas de la agenda del país, en especial en los debates de las regionales donde los candidatos tenían que saber los problemas específicos de ese territorio. 


\begin{tabular}{ccc} 
Candidato & $\begin{array}{c}\text { Índice de profesionalización total } \\
\text { (INPRO) }\end{array}$ & Nivel de profesionalización \\
\hline Duque & 1,44 & Medio-Alto $^{* *}$ \\
\hline Petro & 1,68 & Alto $^{*}$ \\
\hline Fajardo & 1,38 & Medio-Alto $^{* *}$ \\
\hline
\end{tabular}

${ }^{*}$ Nivel Alto $=1 \leq \mathrm{INPRO}<1,5$

${ }^{* *}$ Nivel Medio-Alto $=1,5 \leq \mathrm{INPRO} \leq 2$

Tabla 4. Nivel de profesionalización de las campañas presidenciales en Colombia, 2018 Fuente: Elaboración propia.

Por último, las campañas tuvieron un amplio uso material escrito y de todos los medios técnicos y nuevas tecnologías de comunicación y convergencia. Según el estudio Uso y Apropiación de las TIC en Colombia, 59\% de los colombianos accede a redes sociales al menos 10 veces al día y 70,1\% de los colombianos está en Facebook (Arias, 2017). Lo anterior se evidenció en las campañas de Duque, Petro y Fajardo, donde el uso de las redes sociales, en especial Twitter y Facebook, generó grandes procesos de comunicación transmedia, con propuestas de videos interactivos, foros y fotografías, manejados por grupos de expertos en redes, influenciadores, creación de tendencias, el manejo de la agenda y la interacción permanente con los electores.

De igual manera, se observó el uso de técnicas interactivas para móviles desde el SMS hasta el uso del WhatsApp y telemercadeo, donde se imponían la agenda de medios y se hacía pedagogía electoral. También se identificó en las campañas presidenciales el uso de una nueva herramienta como los drones para tomar fotografías en la plaza pública, ampliando y cerrando los planos para mostrar la magnitud del evento. Finalmente, se debe considerar que los grupos de expertos en manejo de las herramientas técnicas fueron acompañados por los diferentes líderes de sus partidos, líderes de opinión y personajes influyentes en redes sociales y en redes de WhatsApp, alimentado la estrategia de comunicación política de las diferentes campañas.

\section{CONCLUSIÓN}

Este trabajo tuvo como objetivo medir el nivel de profesionalización de las campañas de los tres principales candidatos en la elección presidencial de 2018 en Colombia. En primer lugar, cabe señalar que dos campañas mostraron niveles medio-altos de profesionalización total y una, la de Petro, evidenció un nivel alto. Así, la experiencia acumulada de las campañas electorales de Duque, Petro y 
Fajardo pone de manifiesto que la ventaja en las preferencias electorales no es suficiente para obtener el triunfo, sino que se requieren campañas con cierto nivel de profesionalización.

Asimismo, se evidencia que en la campaña presidencial el nivel de profesionalización se encuentra explicado por la dimensión comunicativa, aspecto que, en cada una de las campañas analizadas, aportó el mayor desarrollo y nivel de cobertura por las propuestas partidarias. Se puede concluir, por lo tanto, que las campañas presidenciales en Colombia siguen dependiendo de las estructuras mediáticas, la proyección de la imagen y la emotividad de la puesta en escena de la herramienta comunicativa. Esto está en contraste con la dimensión organizativa, que muestra que la campaña en Colombia es informal en muchos aspectos. Se pueden tener en cuenta estudios anteriores de profesionalización en Colombia en los que se han evidenciado resultados similares (Restrepo-Echavarría, 2015, 2017; RestrepoEchavarría y Gómez, 2019). Es decir, en contraste con la dimensión organizativa, la parte comunicacional fue la que mayor desarrollo profesional proporcionó en cada una de las contiendas electorales, tanto en las ganadoras como en las perdedoras.

De igual manera, se pudo notar que la dimensión organizativa, que contó con menores índices de desarrollo que la dimensión comunicativa, estuvo regida por las bajas disposiciones partidarias del contexto electoral del país. Como prueba de ello, sus estructuras organizacionales se adaptan con mayor facilidad a los nuevos retos estratégicos, pero fueron campañas que no tuvieron orden ni una cohesión ideológica. En el estudio se evidencia la dependencia de la estructura organizacional de la campaña a la estrategia de comunicación del candidato y a su capacidad administrativa, como fue el caso de las campañas de Petro y Fajardo, cuyo potencial organizacional de la campaña dependió de la personalización y no de un grupo partidista.

Segundo, se puede identificar que, en los procesos electorales en Colombia, es evidente la adaptación de los cimientos estratégicos del país a las dinámicas internacionales. Fenómenos como la personalización de la campaña en el candidato, la implementación de los nuevos discursos, el uso de los medios masivos de comunicación y de las nuevas tecnologías de la información como fuente principal de difusión de propuestas y la estructuración de estrategias alrededor de mensajes emocionales, fueron elementos clave a la hora de diseñar las campañas las tres campañas presidenciales, pues aportaron con mayor efectividad a la hora de contar con el respaldo ciudadano en un entorno polarizado y de baja institucionalidad partidista.

Una tercera reflexión, es la mayor profesionalización de la campaña de Gustavo Petro (INPRO: 1,68), candidato de izquierda que logra histórica votación. Dicho 
nivel devela un cambio sustancial en cómo los partidos y candidatos de izquierda en Colombia han evolucionado en la forma de hacer las campañas electorales, pues las inserciones de técnicas de comunicación política no eran consideradas dentro de estos grupos, que preferían la usanza tradicional de la militancia, la plaza pública y el discurso de masas. Y ahora, candidatos como Petro encuentran en las nuevas tecnologías de la información, los spots, la narrativa y los asesores externos, nuevas formas de cautivar y atraer a los electores. Probablemente el desarrollo profesional en la campaña de un candidato de izquierda haya influido en "el entusiasmo que generó [la] agenda" de Petro (Gamboa, 2019, p. 190), el cual se consolidó en apoyo electoral.

Por último, vale la pena resaltar el aporte de este trabajo en términos analíticometodológicos y empíricos. En este sentido, gracias al índice se obtuvo información empírica para conocer el nivel de profesionalización en que se encuentran las campañas colombianas en suúltimo proceso electoral, y se contribuyó a la generación de nuevo conocimiento al estudio de las campañas electorales en Colombia.

\section{REFERENCIAS}

Arias, C. (2017). Redes sociales, la nueva plaza pública de la campaña presidencial 2018. La rueda de prensa y el comunicado ya están siendo reemplazados por estas plataformas (Social networks, the new public square of the 2018 presidential campaign. The press conference and the statement are already being replaced by these platforms). El Tiempo. Retrieved from https://www.eltiempo.com/politica/gobierno/manejo-de-redes-socialespor-parte-de-los-candidatos-presidenciales-97018

¿A quiénes escuchan Iván Duque y Gustavo Petro? (Who do Iván Duque and Gustavo Petro listen to?). (2018, June 10). El Espectador. Retrieved from https://www.elespectador.com/ noticias/politica/a-quienes-escuchan-ivan-duque-y-gustavo-petro/

Blumler J. G. \& Kavanagh, D. (1999). The third age of political communication: influences and features. Political Communication, 16(3), 209-230. https://doi.org/10.1080/105846099198596

Comitia Consulting. (2018, May 20). Iván Duque - Spot 2 (video). YouTube. Retrieved from https://www.youtube.com/watch?v=0Hk5X8cxTnE .

Cárdenas Ruiz, J. D. (2020). Las elecciones presidenciales del 2018 en Colombia: análisis del cubrimiento noticioso de los noticieros de Caracol y RCN (2018 Presidential elections in Colombia: analysis of the news coverage of Caracol and RCN). Estudios Sobre El Mensaje Periodístico, 26(1), 47-57. https://doi.org/10.5209/esmp.67285

Debates presidenciales, ¿es momento de regularlos? Esta contienda electoral tuvo la paradoja de ver un sinnúmero de debates antes de la primera vuelta, pero ninguno para la segunda (Presidential debates, is it time to regulate them? This electoral contest had the paradox of seeing countless debates before the first round, but none for the second). (2018, June 14). Caracol Radio. Retrieved from https://caracol.com.co/radio/2018/06/14/politica/1528997379_634354.html 
Díaz, O. (2015). Marketing político y profesionalización de las campañas electorales presidenciales del Partido Acción Nacional y del Partido de la Revolución Democrática, 1994-2006 (Political marketing and professionalization of the presidential electoral campaigns of the Partido Acción Nacional and the Partido de la Revolución Democrática, 1994-2006). Polis, 11(1), 119-168.

Directiva unificada No 001 de 2017, de 28 de febrero de 2017, mediante la cual se imparten instrucciones a los servidores públicos, en relación con las jornadas electorales de 2018, para Congreso, Presidente y Vicepresidente de la República (Unified Directive No. 001 of 2017, of February 28, 2017, by means of which instructions are given to public servants, regarding the 2018 election days, for Congress, President and Vice President of the Republic). Procuraduría General de la Nación. Bogotá, Colombia, February 28, 2017, No. 001. Retrieved from https://www.procuraduria.gov.co/relatoria/media/file/flas_ juridico/1640_Directiva\%20Unificada\%20001-2017.pdf.

En Movimiento. Información y prensa. (2018, June 5). Nuevo spot de Gustavo Petro (Gustavo Petro's new spot) (video). YouTube. Retrieved from https://www.youtube.com/watch?v=zedm_Ol3LtE

Farrel, D. (1996). Campaign strategies and tactics. In L. LeDuc, R. G. Niemi, \& P. Norris (Eds.), Comparing democracies: Elections and voting in global perspective (pp. 160 - 183). Thousand Oaks, CA: Sage Publications Ltd.

Gamboa, L. (2019). El reajuste de la derecha colombiana. El éxito electoral del uribismo (The Readjustment of the Colombian Right. Uribismo's Electoral Success). Colombia Internacional, (99), 187-214. https://doi.org/10.7440/colombiaint99.2019.07

Gibson, R. \& Römmele, A. (2001). Changing Campaign Communications: A party-centered theory of professionalized campaigning. Press Politics, 6(4), 31-43. https://doi.org/10.1177/108118001129172323

Gibson, R. \& Römmele, A. (2009). Measuring the professionalization of political campaigning. Party Politics, 15(3), 265-293. https://doi.org/10.1177/1354068809102245

Gómez M. (2018). Iván Duque, una campaña de alto voltaje y finos equilibrios (Iván Duque, a campaign of high voltage and fine balances). El Tiempo. Retrieved from https://www.eltiempo. com/elecciones-colombia-2018/presidenciales/la-campana-presidencial-de-ivan-duque-211060

González, L. \& Restrepo-Echavarría, N. (2020). Campañas presidenciales de 2018 en México: nivel de profesionalización, equipo de campaña y estrategias políticas (Mexican Presidential Campaigns of 2018: Level of Professionalization, War Room, and Political Strategies). América Latina Hoy, 84, 137-161. Retrieved from http://hdl.handle.net/10366/142941

Holtz-Bacha, C. (2002). Campañas electorales en Alemania (Election campaigns in Germany). In F. Priess (Ed.), Relación entre política y medios. Propuestas alemanas en una perspectiva comparada (Relationship between politics and the media. German proposals in a comparative perspective) (pp. 207-229). Buenos Aires, Argentina: Temas Grupo Editorial.

Holtz-Bacha, C. (2007). Professionalization of politics in Germany. In R. M. Negrine, P. Mancine, C. Holtz-Bacha \& S. Papathanassopoulos (Eds.), The professionalization of Political Communication (pp. 63-79). Bristol: Intellect.

Iván Duque presidente 2018-2022. (2018, April 19). Iván Duque Presidente 2018-2022 (Ivan Duque President 2018-2022) (video). YouTube. Retrieved from https://www.youtube.com/watch?v=_rEc_wEpze4 
Julián Duque García. (2018, June 15). El mejor spot publicitario de la campaña de Gustavo Petro (Gustavo Petro's campaign best advertising spot) (video). YouTube. Retrieved from https://www.youtube.com/watch?v=7vd2TzvHCJM\&t=23s

LeDuc, L., Niemi, R. G., \& Norris, P. (2002). Comparing democracies 2: new challenges in the study of elections and voting (Vol. 2). London, UK: Sage.

Liebhart, K. \& Bernhardt, P. (2017). Political Storytelling on Instagram: Key Aspects of Alexander Van der Bellen's Successful 2016 Presidential Election Campaign. Media and Communication, 5(4) 15-25. https://doi.org/10.17645/mac.v5i4.1062

Negrine R., Mancini P., Holtz-Bacha C., \& Papathanassopoulos, S. (Eds). (2007). The Professionalisation of Political Communication. Chicago, IL: The University of Chicago Press.

Nos cogió la noche Cosmovisión. (2018, June 18). Segunda Vuelta de Elecciones Presidenciales 2018 con el Dr. Gilberto Tobón (Second Round of 2018 Presidential Elections with Dr. Gilberto Tobón) (video). YouTube. Retrieved from https://www.youtube.com/watch?v=ZifIMdlaCxQ

Pizarro, E. (2008). Gigantes con pies de barro: los partidos políticos en Colombia (Giants with clay feet: political parties in Colombia). In S. Mainwaring, A. Bejarano, \& E. Pizarro (Eds.), La crisis de la representación democrática en los países andinos (The crisis of democratic representation in the Andean countries) (pp.133-162). Bogota, Colombia: Norma.

Plasser, F. \& Plasser, G. (2002). La Campaña Global: Los nuevos gurúes del Marketing Político en acción (The Global Campaign: The New Political Marketing Gurus in Action). Buenos Aires, Argentina: Temas.

Restrepo-Echavarría, N. J. (2015). La profesionalización de las campañas electorales: las elecciones presidenciales de Colombia 2010 (Professionalization of election campaigns: Colombian presidential elections 2010). Revista Española de Ciencia Política, (38), 85-114. Retrieved from https://recyt.fecyt.es/index.php/recp/article/view/37664

Restrepo-Echavarría, N. J. (2017). La profesionalización de las campañas electorales en Colombia: las elecciones presidenciales en Colombia 1994-2014 (The professionalization of electoral campaigns in Colombia: presidential elections 1994-2014) (Doctoral dissertation). Retrieved from https://eprints.ucm.es/47843/

Restrepo-Echavarría, N. J., Rodríguez, R., \& Castromil, A. R. (2018). Proposal of an indicator to measure the professionalization of election campaigns: The case of Colombia (Proposal of an indicator to measure the professionalization of election campaigns: The case of Colombia). El profesional de la información, 27(2), 289-299. Retrieved from http://profesionaldelainformacion.com/contenidos/2018/mar/07.html

Rocha, F. (2007). La profesionalización de las campañas electorales en Brasil (1989 - 2006) (The professionalization of electoral campaigns in Brazil (1989 - 2006)). (Doctoral dissertation). Retrieved from https://gredos.usal.es/bitstream/handle/10366/18601/ DDPG_Profesionalizacion\%20de\%20las\%20campanas\%20electorales.pdf?sequence $=1$

Sergio Fajardo. (2018, May 24). Alocución del Próximo Presidente de la República, Sergio Fajardo (Address by the Next President of the Republic, Sergio Fajardo) (video). YouTube. Retrieved from https://www.youtube.com/watch?v=mtY-yaR5bvM 
Segrera, M. (n.d.). ¿Quién financió la campaña de Gustavo Petro? (Who funded Gustavo Petro's campaign?). Zoom al billete. Retrieved from https://archivo.colombiacheck.com/ especiales/zoom/Petro

Smith, J. (2004). Campaign Specialists, Party Receptivity, and the Professionalization of Election Campaigns: Theory and a Case Study. Paper presented at the annual meeting of the American Political Science Association, Hilton Chicago and the Palmer House Hilton, Chicago, IL. Retrieved from https://convention2.allacademic.com/one/apsa/apsa04/index. php?click_key=2\&PHPSESSID=1lcsdllfmsjpp5o7kf2rpir5sq

Smith, J. (2009). Campaigning and the Catch-All Party. Party Politics, 15(5), 555-572. https://doi.org/10.1177/1354068809336396

Strömback, J. (2009). Selective Professionalisation of Political Campaigning: A Test of the Party-Centred Theory of Professionalised Campaigning in the Context of the 2006 Swedish Election. Political Studies, 57(1), 95-116. https://doi.org/10.1111/j.1467-9248.2008.00727.x

\section{SOBRE LOS AUTORES}

NÉSTOR JULIÁN RESTREPO ECHAVARRía, doctor en Política, Comunicación y Cultura por la Universidad Complutense de Madrid. Coordinador de la Maestría en Comunicación Política de la Universidad EAFIT. Docente asociado e investigador en la misma universidad. Sus áreas de investigación son comunicación política, opinión pública, movimientos sociales, partidos y sistemas electorales.

(iD) https://orcid.org/0000-0002-2881-1249

LUIS ANTONIO GONZÁLEZ TULE, doctor en Ciencia Política por la Universidad de Salamanca. Profesor asistente en el Departamento de Ciencia Política y Relaciones Internacionales de la Universidad del Norte, de Colombia. Es miembro del Sistema Nacional de Investigadores, nivel I, del CONACYT de México. Sus principales líneas de investigación son instituciones políticas formales e informales y procesos electorales en América Latina.

iD https://orcid.org/0000-0002-3336-8640 